\title{
Microsatellite and minisatellite markers based DNA fingerprinting and genetic diversity of blast and ufra resistant genotypes.
}

\begin{abstract}
A total of 78 alleles and 29 loci were detected from nine microsatellite and three minisatellite markers, respectively across 26 blast and ufra disease resistant genotypes. For blast resistant genotypes, the Polymorphic Information Content (PIC) values ranged from 0.280 to 0.726 and RM21 was considered as the best marker. PIC values ranged from 0.5953 to 0.8296 for ufra resistant genotypes and RM23 was the best marker for characterization of ufra resistant genotypes. The genetic similarity analysis using UPGMA clustering generated nine clusters with coefficient of 0.66 for blast resistant genotypes while five genetic clusters with similarity coefficient of 0.42 for ufra resistant genotypes. In order to develop resistant varieties of two major diseases of rice, hybridisation should be made using the parents, BR29 and NJ70507, BR36 and NJ70507 for blast, while BR11 and Aokazi, BR3 and Aokazi, Rayda and BR3 and Rayda and BR11 for ufra.
\end{abstract}

Keyword: Biodiversity; Blast and ufra diseases; Resistant genotypes; Rice. 